\title{
Burnside-Brauer Theorem for Table Algebras
}

\author{
J. Bagherian \\ Department of Mathematics \\ University of Isfahan \\ P.O. Box 81746-73441 \\ Isfahan, Iran \\ bagherian@sci.ui.ac.ir
}

\author{
A. Rahnamai Barghi * \\ Department of Mathematics \\ K. N. Toosi University of Technology \\ P.O. Box 16315-1618 \\ Tehran, Iran \\ rahnama@kntu.ac.ir
}

Submitted: Jul 25, 2011; Accepted: Sep 30, 2011; Published: Oct 17, 2011

Mathematics Subject Classification: 16S99, 16G30

\begin{abstract}
In the character theory of finite groups the Burnside-Brauer Theorem is a wellknown result which deals with products of characters in finite groups. In this paper, we first define the character products for table algebras and then by observing the relationship between the characters of a table algebra and the characters of its quotient, we provide a condition in which the products of characters of table algebras are characters. As a main result we state and prove the Burnside-Brauer Theorem on finite groups for table algebras.
\end{abstract}

\section{Introduction}

One of important results in the character theory of finite groups is the Burnside-Brauer Theorem. This theorem is as the following:

Theorem 1.1. (Burnside-Brauer) Let $\chi$ be a faithful character of a finite group $G$ and suppose that $\chi(g)$ takes on exactly $k$ different values for $g \in G$. Then every $\psi \in \operatorname{Irr}(G)$ is a constituent of one the characters $\chi^{i}, 0 \leq i<k$.

The main result in this paper is to state and prove an analog of the Burnside-Brauer Theorem for table algebras. Therefore, we deal with products of characters in table algebras. We mention that products of characters in table algebras need not be characters in general. In order to provide a condition in which the products of characters of a given table algebra are characters, we need to observe the relationship between the characters of a table algebra and the characters of its quotient. In section 2, we show that for a

*corresponding author 
table algebra $(A, B)$ and a strongly normal closed subset $C$ of $B$, the set of irreducible characters of $(A / C, B / C)$ can be consider as a subset of irreducible characters of $(A, B)$.

An interesting problem on characters of table algebras is character products. Since table algebras are not Hopf algebras in general, character products need not be characters. In section 3, we define the character products for table algebras and we obtain a condition in which the character products are characters. Finally, we prove the Burnside-Brauer Theorem for table algebras which is a well know theorem in the theory of finite groups.

Throughout this paper we follow from [1] for the definition of table algebras and related notions. Hence we deal with non-commutative table algebras as the following:

A table algebra $(A, B)$ is a finite dimensional algebra $A$ over the complex field $\mathbb{C}$ and a distinguished basis $B=\left\{b_{1}=1_{A}, \cdots, b_{d}\right\}$ for $A$, where $1_{A}$ is a unit, such that the following properties hold:

(I) The structure constants of $B$ are nonnegative real numbers, i.e., for $a, b \in B$ :

$$
a b=\sum_{c \in B} \lambda_{a b c} c, \quad \lambda_{a b c} \in \mathbb{R}^{+} \cup\{0\} .
$$

(II) There is a semilinear involutory anti-automorphism (denoted by ${ }^{*}$ ) of $A$ such that $B^{*}=B$.

(III) For all $a, b \in B, \lambda_{a b 1_{A}}=0$ if $b \neq a^{*}$; and $\lambda_{a a^{*} 1_{A}}>0$.

Remark 1.2. (i) Let $(A, B)$ be a table algebra. Then [1, Theorem 3.11] implies that $A$ is semisimple.

(ii) For any table algebra $(A, B)$, there is a unique algebra homomorphism $||:. A \rightarrow \mathbb{C}$, called degree map, such that $|b|=\left|b^{*}\right|>0$ for all $b \in B$ (see [1, Theorem 3.14]).

(iii) If for all $b \in B,|b|=\lambda_{b b^{*} 1_{A}}$, then the table algebra $(A, B)$ is called standard table algebra.

(iv) Let $(A, B)$ be a table algebra. Let $B^{\prime}=\left\{\lambda_{b} b \mid b \in B\right\}$, where $\lambda_{1_{A}}=1$ and $\lambda_{b}=\lambda_{b^{*}} \in$ $\mathbb{R}^{+}$for all $b \in B$. Then $\left(A, B^{\prime}\right)$ is also a table algebra which is called a rescaling of $(A, B)$ (see [1, Section 3]). If $\left(A, B^{\prime}\right)$ is a rescaling of $(A, B)$ then we may simply say that $B^{\prime}$ is a rescaling of $B$. From [1, Theorem 3.15] one can see that any table algebra can be rescaled to a standard table algebra.

The value $|b|$ is called the degree of the basis element $b$. For an arbitrary element $\sum_{b \in B} x_{b} b \in A$, we have $\left|\sum_{b \in B} x_{b} b\right|=\sum_{b \in B} x_{b}|b|$.

For each $a=\sum_{b \in B} x_{b} b$, we set $a^{*}=\sum_{b \in B} \overline{x_{b}} b^{*}$, where $\overline{x_{b}}$ means the complex conjugate of $x_{b}$. For any $x=\sum_{b \in B} x_{b} b \in A$, denote by $\operatorname{Supp}(x)$ as the set of all basis elements $b \in B$ such that $x_{b} \neq 0$. If $E, D \subseteq B$, then we set $E D=\bigcup_{e \in E, d \in D} \operatorname{Supp}(e d)$. 
A nonempty subset $C \subseteq B$ is called a closed subset, if $C^{*} C \subseteq C$. We denote by $\mathcal{C}(B)$ the set of all closed subsets of $B$. In addition, $C \in \mathcal{C}(B)$ is said to be normal in $B$ if $b C=C b$ for every $b \in B$, and denote it by $C \unlhd B$.

Throughout the paper, we focus on standard table algebras, although our main result (Theorem 3.7) is valid for an arbitrary table algebra.

\section{Characters of quotient table algebras}

Let $(A, B)$ be a table algebra with basis $B$ and let $C \in \mathcal{C}(B)$. From [1, Proposition 4.7], it follows that $\{C b C \mid b \in B\}$ is a partition of $B$. A subset $C b C$ is called a $C$-double coset or double coset with respect to the closed subset $C$. Let

$$
b / C:=\left|C^{+}\right|^{-1}(C b C)^{+}=\left|C^{+}\right|^{-1} \sum_{x \in C b C} x
$$

where $C^{+}=\sum_{c \in C} c$ and $\left|C^{+}\right|=\sum_{c \in C}|c|$. Then the following theorem is an immediate consequence of [1, Theorem 4.9]:

Theorem 2.1. Let $(A, B)$ be a table algebra and let $C \in \mathcal{C}(B)$. Suppose that $\left\{b_{1}=\right.$ $\left.1_{A}, \ldots, b_{k}\right\}$ be a complete set of representatives of $C$-double cosets. Then the vector space spanned by the elements $b_{i} / C, 1 \leq i \leq k$, is a table algebra ( which is denoted by $A / C$ ) with a distinguished basis $B / C=\left\{b_{i} / C \mid 1 \leq i \leq k\right\}$. The structure constants of this algebra are given by the following formula:

$$
\gamma_{i j k}=\left|C^{+}\right|^{-1} \sum_{r \in C b_{i} C, s \in C b_{j} C} \lambda_{r s t}
$$

where $t \in C b_{k} C$ is an arbitrary element.

The table algebra $(A / C, B / C)$ is called the quotient table algebra of $(A, B)$ modulo $C$.

Let $(A, B)$ be a table algebra and $C \in \mathcal{C}(B)$. Set $e=\left|C^{+}\right|^{-1} C^{+}$. Then $e$ is an idempotent for the table algebra $A$ and the subalgebra $e A e$ is equal to the quotient table algebra $(A / C, B / C)$ modulo $C$, see $[1]$.

The proof of the following theorem is given in [8, Theorem 2.1].

Theorem 2.2. Let $C \unlhd B$. Then the map $\pi: A \rightarrow A / C$ defined by $\pi(b)=\frac{|b|}{|b / C|}(b / C)$ is an algebra homomorphism.

Theorem 2.3. Let $C \unlhd B$ and let $\psi: A / C \rightarrow \operatorname{Mat}_{\mathrm{s}}(\mathbb{C})$ be a representation of $A / C$. Then $\bar{\psi}: A \rightarrow \operatorname{Mat}_{\mathrm{s}}(\mathbb{C})$ defined by $\bar{\psi}(b)=\frac{|b|}{|b / C|} \psi(b / C)$ is a representation of $A$.

Proof. Put $\bar{\psi}=\psi \circ \pi$, where $\pi$ is defined in Theorem 2.2. Then $\bar{\psi}$ is an algebra homomorphism and $\bar{\psi}(b)=\psi \circ \pi(b)=\psi\left(\frac{|b|}{|b / C|} b / C\right)=\frac{|b|}{|b / C|} \psi(b / C)$, as desired. 
Remark 2.4. By Theorem 2.3 we may embed $\operatorname{Irr}(A / C)$ into $\operatorname{Irr}(A)$, indeed if $\chi$ and $\psi$ are distinct characters of $A / C$, then $\chi(b / C) \neq \psi(b / C)$, for some $b / C \in B / C$ and so $\bar{\chi}(b) \neq \bar{\psi}(b)$, where $\bar{\chi}$ is a the character of $A$ defined by $\bar{\chi}(b)=\frac{|b|}{|b / C|} \chi(b / C)$. Other proof for embedding of $\operatorname{Irr}(A / C)$ into $\operatorname{Irr}(A)$ can be found in [8].

In the following we consider another property on $C$ which is stronger than normality, namely strongly normal closed subset. The rest of this section deals with the quotient of table algebras modulo $C$, where $C$ is a strongly normal closed subset.

Definition 2.5. A closed subset $C$ of $B$ is said to be strongly normal and denoted by $C \unlhd^{\prime} B$, if for each $b \in B$

$$
b^{*} C b \subseteq C .
$$

In the following we show that a strongly normal closed subset is a normal closed subset.

Lemma 2.6. Every strongly normal closed subset is a normal closed subset.

Proof. Let $C \unlhd^{\prime} B$. Then for $b \in B$ we have $b^{*} C b \subseteq C$, and so $b C b^{*} C b \subseteq b C$ which implies that $C b \subseteq b C$. On the other hand, from $b C b^{*} \subseteq C$ it follows that $b C b^{*} C b \subseteq C b$, and so $b C \subseteq C b$. Thus $C b=b C$, as desired.

The following example shows that the converse of the above lemma is not true, i.e., a normal closed subset is not necessarily strongly normal closed subset.

Example 2.7. Let $q \geq 2$ and $B=\left\{r_{0}, r_{1}, \ldots, r_{q+1}\right\}$ be a basis for a complex vector space A of dimension $q+2$. We define multiplication

$$
r_{i} r_{j}= \begin{cases}(q-1) r_{0}+(q-2) r_{i}, & \text { if } i=j \neq 0 \\ \sum_{k \neq 0, i, j} r_{k}, & \text { if } i \neq j\end{cases}
$$

for all $i, j$ with $1 \leq i, j \leq q+1$, and $r_{i} r_{0}=r_{i}$ for all $i$. This extends to a multiplication in $A$ which is commutative and association with unit element $r_{0}=1_{A}$. A direct computation shows that $(A, B)$ is a table algebra where $r_{i}^{*}=r_{i}$ for all $i$; and $\left|r_{i}\right|=q-1$ for $i>0$. Clearly the set $\left\{r_{0}, r_{i}\right\}$ for every $i \neq 0$ is a normal closed subset but it is not a strongly normal closed subset. In fact, the construction of this table algebra is given in [7].

Theorem 2.8. Let $b \in B$ and $C \in \mathcal{C}(B)$. Then $|b / C|=1$ if and only if $b^{*} C b \subseteq C$.

Proof. Let $T=\left\{b_{1}=1_{A}, b_{2}, \ldots, b_{t}\right\}$ be a complete set of representatives of $C$-double cosets and let $b=b_{i}$ for some $i, 1 \leq i \leq t$.

Suppose that $|b / C|=1$ and let $d \in b^{*} C b$. Since $1_{A} \in C$, we have $d \in\left(C b^{*} C\right)(C b C)$. Then there exists $r \in C b^{*} C$ and $s \in C b C$ such that $\lambda_{r s d} \neq 0$. As $B=\bigcup_{j=1}^{t} C b_{j} C$, we may assume that $d \in C b_{k} C$ for some $k, \quad 1 \leq k \leq t$. So $\gamma_{i^{*} i k} \neq 0$ where

$$
\gamma_{i^{*} i k}=\left|C^{+}\right|^{-1} \sum_{r \in C b_{i}{ }^{*} C, s \in C b_{i} C} \lambda_{r s d} .
$$


But from the assumption we conclude that $(b / C)\left(b^{*} / C\right)=\left\{1_{A} / C\right\}$. Hence $\gamma_{i^{*} i 1} \neq 0$ and so that $k=1$. Thus $d \in C$ and so $b^{*} C b \subseteq C$.

Conversely, let $b^{*} C b \subseteq C$. Then $\left(C b^{*} C\right)(C b C) \subseteq C$ and so

$$
\gamma_{i^{*} i 1} \leqslant\left|C^{+}\right|^{-1} \sum_{r, s \in C} \lambda_{r s d}
$$

for $d \in C$. Now from (1) and the equalities $\sum_{r, s \in C} \lambda_{r s d}=\sum_{r \in C}|r|=\left|C^{+}\right|$(see [1, Proposition 2.3(i)]) we deduce that $\gamma_{i^{*} i 1}=1$. Thus $|b / C|=1$, as desired.

Corollary 2.9. Let $(A, B)$ be a table algebra and $C \in \mathcal{C}(B)$. Then $(A / C, B / C)$ is a group algebra if and only if $C \unlhd^{\prime} B$.

Proof. This follows immediately from Theorem 2.8.

Corollary 2.10. Let $C \unlhd^{\prime} B$ and $\psi$ be a representation of $A / C$. Then the mapping $\bar{\psi}: b \mapsto|b| \psi(b / C)$ for every $b \in B$, is a representation for $A$.

Proof. This is a direct consequence of Theorem 2.3 and Theorem 2.8.

\section{Character products}

For an associative algebra A, the tensor product $V \otimes W$ of two $A$-modules $V$ and $W$ is a vector space, but not necessarily an $A$-module. In order to make an $A$-module on $V \otimes W$, there must be a linear binary operation $\Delta: A \rightarrow A \otimes A$ which is also an algebra homomorphism. This is an important property for the algebra $A$ becomes a Hopf algebra. For instance, in group theory the tensor products of two $G$-modules $V$ and $W$ gives us a module, indeed the group algebra $\mathbb{C} G$ is a Hopf algebra with $\Delta: g \rightarrow g \otimes g$. So if $\chi$ and $\psi$ are afforded by two $G$-modules, then their tensor product affords the character $\chi \psi(g):=\chi(g) \psi(g)$ which is called the character product of $\chi$ and $\psi$.

In general, a table algebra $(A, B)$ is not a Hopf algebra and so it is not generally possible to define the structure of an $A$-module on $V \otimes W$. In [2] Doi introduced a generalization of Hopf algebras and defined a binary linear operation $\Delta: b \rightarrow \frac{1}{|b|} b \otimes b$, $b \in B$. By considering this binary linear operation, we define the character product of $\chi$ and $\psi$ by:

$$
\chi \psi(b):=\frac{1}{|b|} \chi(b) \psi(b), \quad b \in B .
$$

Since $\Delta$ is not necessarily an algebra homomorphism, a character product in a table algebra is not generally a character. It might be mentioned that this is an analog of association schemes which has already done by Hanaki in [4]. 
Remark 3.1. Let $(A, B)$ be a table algebra and let $B^{\prime}=\left\{\lambda_{b} b \mid b \in B\right\}$ be a rescaling of $B$. Since for every $\chi, \psi \in \operatorname{Irr}(A)$ and $b^{\prime}=\lambda_{b} b \in B^{\prime}$,

$$
\chi \psi\left(b^{\prime}\right)=\chi \psi\left(\lambda_{b} b\right)=\lambda_{b} \chi \psi(b)=\lambda_{b} \frac{1}{|b|} \chi(b) \psi(b)=\frac{1}{\left|b^{\prime}\right|} \chi\left(b^{\prime}\right) \psi\left(b^{\prime}\right),
$$

it follows that the rescaling of table algebras preserves the character products.

In this section we will give a condition in which the character products are characters. We state a fact to use it later.

Lemma 3.2. ([8, Lemma 3.4]) Let $(A, B)$ be a table algebra, $C$ a closed subset of $B$, and $e=\left|C^{+}\right|^{-1} C^{+}$. Let $\chi$ be a character of $A$ afforded by an $A$-module $V$. Then the following are equivalent:

(1) $\operatorname{dim}_{\mathbb{C}}(e V) \neq 0$,

(2) $e V=V$,

(3) $\chi(e) \neq 0$,

(4) $\chi(e)=\chi(1)$,

(5) $\chi(a)=\chi(e a e), \forall a \in A$.

Throughout this section we assume that $(A, B)$ is a table algebra with a strongly normal closed subset $C$ and $e=\left|C^{+}\right|{ }^{-1} C^{+}$.

Let $V$ and $W$ be $A / C$-module and $A$-module, respectively. We define a multiplication of $A$ on $V \otimes W$ as the following:

$$
b(v \otimes w):=(b / C) v \otimes b w, \quad v \in V, w \in W, b \in B .
$$

Lemma 3.3. Let $V$ be an irreducible $A$-module with $\operatorname{dim}_{\mathbb{C}}(e V) \neq 0$ and let $W$ be an A-module. Then $V \otimes W$ is an A-module given by the multiplication in (3).

Proof. We first claim that $\mu: A \rightarrow A / C \otimes A$ by $\mu(b)=b / C \otimes b$, for any $b \in B$ is an algebra homomorphism. Let $b, c \in B$ be given. Then

$$
\mu(b c)=\mu\left(\sum_{d \in B} \lambda_{b c d} d\right)=\sum_{d \in B} \lambda_{b c d} \mu(d)=\sum_{d \in B} \lambda_{b c d} d / C \otimes d .
$$

On the other hand,

$$
\mu(b) \mu(c)=(b / C \otimes b)(c / C \otimes c)=\sum_{d \in B} \lambda_{b c d}(b / C)(c / C) \otimes d .
$$

Now in the above equality, if $\lambda_{b c d} \neq 0$ then $\gamma_{b / C, c / C, d / C} \neq 0$ and so $(b / C)(c / C)=d / C$, indeed by Corollary $2.9 B / C$ is a group. Thus $\mu(b) \mu(c)=\sum_{d \in B} \lambda_{b c d} d / C \otimes d$ which is equal to $\mu(b c)$ by $(4)$, and the claim is proved. Since $\operatorname{dim}_{\mathbb{C}}(e V) \neq 0$, then from Lemma 3.2 it follows that $V$ is an $A / C$-module. This implies that $b(v \otimes w)=\mu(b)(v \otimes w)$, and the proof is complete. 
Lemma 3.4. Let $\chi$ be an irreducible character afforded by $A$-module $V$ such that $\operatorname{dim}_{\mathbb{C}}(e V)$ $\neq 0$. Then for every $A$-module $W$ the tensor product $V \otimes W$ is an $A$-module which affords the character $\chi \psi$, where $\psi$ is a character of $A$ afforded by $W$.

Proof. From [8, Lemma 3.7] it follows that $e V$ is an $A$-module and so irreducibility of $V$ implies that $V=e V \neq 0$. Therefore, Lemma 3.2 implies that $\chi(a)=\chi(e a e)$, for $a \in A$. This equality, together with $e b e=\frac{|b|}{|b / C|}(b / C)$ for $b \in B$, implies that $\chi(b / C)=\frac{1}{|b|} \chi(b)$, for $b \in B$, here we used the fact that $|b / C|=1$ by Theorem 2.8 since $C$ is a strongly normal closed subset of $B$.

From Lemma 3.2 and Lemma 3.3 it follows that $V \otimes W$ is an $A$-module and $V$ can be considered as an $A / C$-module, respectively. Now let $D_{1}: A / C \rightarrow$ Mat $_{d_{1}}(\mathbb{C})$ and $D_{2}: A \rightarrow$ $\operatorname{Mat}_{d_{2}}(\mathbb{C})$ be representations of $A / C$ and $A$ corresponding to $V$ and $W$, respectively. Then $\left(D_{1} \otimes D_{2}\right) \circ \mu: A \rightarrow \operatorname{Mat}_{\mathrm{d}_{1} \mathrm{~d}_{2}}(\mathbb{C})$ is a representation of $A$ corresponding to $V \otimes W$, where $\mu: A \rightarrow A / C \otimes A$ is the algebra homomorphism given in the proof of Lemma 3.3. The argument in the preceding paragraph shows that the character afforded by $D_{1}$ is $\chi$. Now we have $\left(\left(D_{1} \otimes D_{2}\right) \circ \mu\right)(b)=D_{1}(b / C) \otimes D_{2}(b)$, so the character is $\chi(b / C) \psi(b)=\frac{1}{|b|} \chi(b) \psi(b)$, for $b \in B$, and we are done.

Theorem 3.5. Let $\chi$ be an irreducible character of $A$ such that $\chi(e) \neq 0$. Then $\chi \psi$ is a character of $A$, where $\psi$ is a character of $A$.

Proof. Let $V$ be an irreducible $A$-module which affords the character $\chi$. From $\chi(e) \neq 0$ we have $e V \neq 0$. Now the result follows from Lemma 3.4.

Theorem 3.6. Let $\chi, \psi \in \operatorname{Irr}(A)$. If $\chi(e)=1$, then $\chi \psi \in \operatorname{Irr}(A)$.

Proof. Let $V$ and $W$ be two irreducible $A$-modules which afford $\chi$ and $\psi$ respectively. The equality $\chi(e)=1$ implies that $\operatorname{dim}_{\mathbb{C}}(V)=\chi(e)=1$. From Lemma 3.2 and Theorem 3.5 it follows that $\chi$ is a linear character of $A / C$ and $\chi \psi$ is a character, respectively. Moreover, $\operatorname{dim}_{\mathbb{C}}(V)=1$ implies that every $A$-submodule of $V \otimes W$ is of the form $V \otimes W^{\prime}$ where $W^{\prime}$ is an $A$-submodule of $W$. Therefore, by irreducibility of $W$ the $A$-module $V \otimes W$ is irreducible and so $\chi \psi \in \operatorname{Irr}(A)$. This completes the proof.

Let $A$ be a finite dimensional algebra with a basis $w_{1}, \ldots, w_{r}$ over a field $F$. Let $\zeta$ be a non-degenerate feasible trace on $A$. Then from $[6], \zeta$ induces a dual form $[\cdot, \cdot]$ on $\operatorname{Hom}_{F}(A, F)$ in which for every $\chi, \varphi \in \operatorname{Hom}_{F}(A, F)$ we have

$$
[\chi, \varphi]=\sum_{i=1}^{r} \chi\left(w_{i}\right) \varphi\left(\hat{w}_{i}\right)
$$

where $\hat{w}_{1}, \ldots, \hat{w}_{r}$ is the dual basis defined by $\zeta\left(w_{i} \hat{w}_{j}\right)=\delta_{i, j}$.

Now let $(A, B)$ be a table algebra. Define a linear function $\zeta$ on $A$ defined by $\zeta(b)=$ $\delta_{b, 1_{A}}\left|B^{+}\right|$, for $b \in B$. Then $\zeta$ is a non-degenerate feasible trace on $A$ and it follows that the dual form $[\cdot, \cdot]$ on $\operatorname{Hom}_{\mathbb{C}}(A, \mathbb{C})$ is as follows:

$$
[\chi, \varphi]=\frac{1}{\left|B^{+}\right|} \sum_{b \in B} \frac{1}{|b|} \chi(b) \varphi\left(b^{*}\right)
$$


for every $\chi, \varphi \in \operatorname{Hom}_{\mathbb{C}}(A, \mathbb{C})$. From $[6$, Section 5], one can see that $[\chi, \psi]=0$, for any $\chi, \psi \in \operatorname{Irr}(A)$, where $\chi \neq \psi$.

Let $\chi$ be a character of table algebra $(A, B)$. The following subset of $B$

$$
K(\chi)=\{b \in B: \chi(b)=|b| \chi(1)\}
$$

is a closed subset of $B$. The proof of this result can be found in [3, Theorem 3.2] for the case of association scheme, but the proof also works for table algebras.

Now we can state the main theorem.

Theorem 3.7. Let $(A, B)$ be a table algebra. Suppose that $A$ has a character $\chi$ with $K(\chi)=\left\{1_{A}\right\}$ such that $\chi(b) /|b|$ takes on exactly $k$ different values for $b \in B$. Then, for each irreducible character $\psi$ of $A$, there exists $0 \leq i \leq k-1$ such that $\left[\chi^{i}, \psi\right] \neq 0$.

Proof. Let $\alpha_{1}, \ldots, \alpha_{k}$ be the distinct values taken by $\chi(b) /|b|, b \in B$. Define $B_{t}=$ $\left\{b \in B|\chi(b)=| b \mid \alpha_{t}\right\}$. Assume that $\alpha_{1}=\chi(1)$ so that $B_{1}=K(\chi)$. Fix $\psi \in \operatorname{Irr}(A)$ and let $\beta_{i}=\sum_{b \in B_{i}} \psi\left(b^{*}\right)$ for $1 \leq i \leq k$. Since $\chi^{2}(b)=\frac{1}{|b|} \chi(b)^{2}$, it follows that $\chi^{j}(b)=\frac{1}{|b|^{j-1}} \chi(b)^{j}$. Hence

$$
\begin{aligned}
{\left[\chi^{j}, \psi\right] } & =\frac{1}{\left|B^{+}\right|} \sum_{b \in B} \frac{1}{|b|} \chi^{j}(b) \psi\left(b^{*}\right) \\
& =\frac{1}{\left|B^{+}\right|} \sum_{i=1}^{k} \sum_{b \in B_{i}} \frac{1}{|b|^{j}} \chi(b)^{j} \psi\left(b^{*}\right) \\
& =\frac{1}{\left|B^{+}\right|} \sum_{i=1}^{k}\left(\alpha_{i}\right)^{j} \beta_{i} .
\end{aligned}
$$

Therefore, if $\left[\chi^{j}, \psi\right]=0$ for all $0 \leq j \leq k-1$, then

$$
\sum_{i=1}^{k}\left(\alpha_{i}\right)^{j} \beta_{i}=0, \quad j=0,1, \ldots, k-1 .
$$

Let $M:=\left(a_{i, j}\right)$ be a $k \times k$ matrix whose $i$ th row and $j$ th column is $\left(\alpha_{i}\right)^{j}$ and let $X=\left(\beta_{1}, \beta_{2}, \ldots, \beta_{k}\right)$. Therefore (6) shows that $X M=0$. But the determinant of $M$ is Vandermonde determinant and is equal to $\pm \Pi_{i<j}\left(\alpha_{i}-\alpha_{j}\right) \neq 0$. It follows that $X=0$. But $\beta_{1}=\psi\left(1_{A}\right) \neq 0$, which is a contradiction. This completes the proof of the theorem.

Remark 3.8. Let $(A, B)$ be a table algebra. If $(A, B)$ is commutative or isomorphic to the adjacency algebra of a group-like scheme, then for every $\chi \in \operatorname{Irr}(A), \chi^{i}$ is a linear combination of $\operatorname{Irr}(A)$ (see [5, Theorem 4.1]). Suppose that $\chi^{i}=\sum_{\varphi \in \operatorname{Irr}(A)} \lambda_{\varphi}^{(i)} \varphi$, where $\lambda_{\varphi}^{(i)} \in \mathbb{C}$. Then it follows from Theorem 3.7 that for every $\psi \in \operatorname{Irr}(A)$, there exists $0 \leq i \leq k-1$ such that $\lambda_{\psi}^{(i)} \neq 0$. 
The following corollary is an analog of the Burnside-Brauer Theorem on finite groups for table algebras.

Corollary 3.9. Let $(A, B)$ be a table algebra. Suppose that $A$ has a character $\chi$ with $K(\chi)=\left\{1_{A}\right\}$ such that $\chi(b) /|b|$ takes on exactly $k$ different values for $b \in B$. If all powers of $\chi$ by itself is a character, then each irreducible character of $A$ appears as an irreducible component of one of $\chi^{i}$, where $0 \leq i \leq k-1$.

Proof. This follows immediately from Theorem 3.7.

Remark 3.10. By using Theorem 3.9 for $(A, B)=(\mathbb{C} G, G)$, where $G$ is a finite group, we get the Burnside-Brauer Theorem on finite groups (see Theorem 1.1).

Examples 3.11 and 3.12 below give table algebras which satisfy the assumptions of Corollary 3.9.

Example 3.11. Let $A$ be a $\mathbb{C}$-linear space with the basis $B=\left\{1_{A}, b, c\right\}$ such that

$$
\begin{aligned}
b^{2} & =21_{A} \\
c^{2} & =\frac{1}{2} 1_{A}+\frac{1}{2} b \\
b c & =c b=c
\end{aligned}
$$

Then one can see that the pair $(A, B)$ is a table algebra and an easy computation shows that the character table of $(A, B)$ is as follows:

\begin{tabular}{c|ccc} 
& $1_{A}$ & $b$ & $c$ \\
\hline$\chi_{1}$ & 1 & 1 & $\frac{1}{2}$ \\
$\chi_{2}$ & 1 & 1 & $-\frac{1}{2}$ \\
$\chi_{3}$ & 1 & -1 & 0
\end{tabular}

Put $\chi=2 \chi_{3}$. Then it is easy to see that $\chi$ is a character of $A$ such that $K(\chi)=\left\{1_{A}\right\}$. Moreover, $\chi^{2}=2 \chi_{1}+2 \chi_{2}$ and all powers of $\chi$ by itself is a character.

Example 3.12. Let $A$ be a $\mathbb{C}$-linear space with the base $B=\left\{b_{0}=1_{A}, b_{1}, b_{2}, b_{3}, b_{4}\right\}$ such that

$$
\begin{aligned}
b_{1}^{2} & =b_{0}, \\
b_{i}^{2} & =2 b_{0}+b_{i}, \\
b_{1} b_{i} & =b_{i} b_{1}=b_{i}, \\
b_{2} b_{3} & =b_{3} b_{2}=2 b_{4}, \\
b_{2} b_{4} & =b_{4} b_{2}=2 b_{3}, \\
b_{3} b_{4} & =b_{4} b_{3}=2 b_{2},
\end{aligned}
$$


where $i \in\{2,3,4\}$. Then it is easy to check that $(A, B)$ is a table algebra. A simple computation shows that the character table of the algebra $A$ is

\begin{tabular}{c|ccccc} 
& $b_{0}$ & $b_{1}$ & $b_{2}$ & $b_{3}$ & $b_{4}$ \\
\hline$\chi_{1}$ & 1 & 1 & 2 & 2 & 2 \\
$\chi_{2}$ & 1 & 1 & 2 & -2 & -2 \\
$\chi_{3}$ & 1 & 1 & -2 & 2 & -2 \\
$\chi_{4}$ & 1 & 1 & -2 & -2 & 2 \\
$\chi_{5}$ & 1 & -1 & 0 & 0 & 0
\end{tabular}

If we put $\chi=4 \chi_{5}$, then clearly $\chi$ is a character of $G$ such that $K(\chi)=\left\{1_{A}\right\}$. Furthermore, one can see that $\chi^{2}=4 \chi_{1}+4 \chi_{2}+4 \chi_{3}+4 \chi_{4}$ and all powers of $\chi$ by itself is a character.

\section{ACKNOWLEDGMENTS}

The authors are very grateful to the anonymous referee for his careful reading and valuable comments and suggestions.

\section{References}

[1] Z. Arad, E. Fisman, M. Muzychuk, Generalized Table Algebras, Israel J. Math. 114 (1999) 29-60.

[2] Y.Doi, Bi-Frobenius Algebras and Group-Like Algebras, Hopf algebras in: Lecture Note in Pure and Appl.Math, Vol. 237, Dekker, New York, pp.143-155, 2004.

[3] A. Hanaki, Characters of Association Schemes and Normal Closed Subsets, Graphs Combin. 19 (2003) 363-369.

[4] A. Hanaki, Character Products of Association Schemes, J. Algebra 283 (2005) 596-603.

[5] A. Hanaki, Nilpotent Schemes and Group-Like Schemes, J. Combin. Theory Ser. A 115 (2008) 226-236.

[6] D. G. Higman, Coherent Algebras, J. Linear Algebra and it's Applications 93 (1987) 209-239.

[7] I. Ponomarenko, A. Rahnamai Barghi, On Amorphic C-algebras, Journal of Mathematical Sciences, Vol. 145 No. 3 (2007).

[8] Bangteng Xu, Characters of Table Algebras and Applications to Association Schemes, J. Combin. Theory Ser. A 115 (2008) 1358-1373. 